\title{
Spectral properties and a Parseval's equality in the singular case for $q$-Dirac problem
}

\section{Seyfollah Mosazadeh ${ }^{1 *}$ (1)}

\section{${ }^{*}$ Correspondence:}

s.mosazadeh@kashanu.ac.ir

'Department of Pure Mathematics, Faculty of Mathematical Sciences,

University of Kashan, Kashan, Iran

\section{Springer}

\begin{abstract}
This paper is devoted to studying a $q$-analog of the singular Dirac problem. First, we investigate some spectral properties of the problem. Then we prove the existence of a spectral function and establish a Parseval's equality, for the singular q-Dirac system in a Hilbert space. Although there were given some results for this type of problem, we think that Parseval's equality has not been studied yet.
\end{abstract}

MSC: 39A13; 34L40; 34L05; 33D15

Keywords: $q$-Dirac problem; singularity; spectral function; Parseval's equality

\section{Introduction}

In 1910, Jackson introduced the $q$-derivative operator, $D_{q}$, different from the classical derivative, and its right-inverse, the $q$-integration $[19,20]$. Then the $q$-calculus was based on these notations. This calculus has a lot of applications in different mathematical areas, such as calculus of variations, orthogonal polynomials, theory of relativity, quantum theory and statistical physics (see [1, 23, 29]). Furthermore, there are several physical models involving $q$-functions, $q$-derivatives, $q$-integrals and their related problems $[9,11,12,15,28]$.

In the present paper, we study an analog of Dirac system when the differential operator is replaced by Jackson's $q$-difference operator $D_{q}$ (definitions are given in the next section). Let us consider the $q$-problem which consists of the $q$-Dirac system

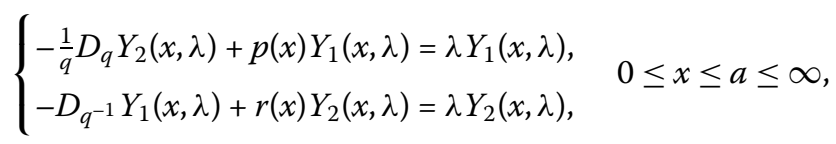

and the boundary conditions

$$
\begin{aligned}
& Y_{1}(0, \lambda)=Y_{2}(0, \lambda), \\
& Y_{1}\left(q^{-n-1}, \lambda\right)=Y_{2}\left(q^{-n}, \lambda\right), \quad n \in \mathbb{N},
\end{aligned}
$$

where $\lambda$ is the spectral parameter, $q \in(0,1)$ is fixed, $p(x)$ and $r(x)$ are real-valued functions and continuous at zero, and $p(x), r(x) \in L_{q}^{1}(0, \infty)$.

(c) The Author(s) 2019. This article is licensed under a Creative Commons Attribution 4.0 International License, which permits use, sharing, adaptation, distribution and reproduction in any medium or format, as long as you give appropriate credit to the original author(s) and the source, provide a link to the Creative Commons licence, and indicate if changes were made. The images or other third party material in this article are included in the article's Creative Commons licence, unless indicated otherwise in a credit line to the material. If material is not included in the article's Creative Commons licence and your intended use is not permitted by statutory regulation or exceeds the permitted use, you will need to obtain permission directly from the copyright holder. To view a copy of this licence, visit http://creativecommons.org/licenses/by/4.0/. 
The limit-point and limit-circle classifications of the singular point, $x=\infty$ of the $q$ difference equation were defined in [8], and using Titchmarsh's technique, sufficient conditions that the singular point is in a limit-point case were given. In the case when $q=1$, i.e. $D_{q}=\frac{d}{d x}$, the Dirac system (1.1) was studied in many works, and for more details we refer the reader to Refs. $[10,14,16,22,25-27,30]$. In [2, 3], a $q$-analog of one dimensional Dirac system on a finite interval was investigated and the authors studied the existence and uniqueness of its solution, and some spectral properties. Also, the asymptotic formulas for the eigenvalues and the eigenfunctions were obtained in [18].

The paper is organized as follows. In Sect. 2, we introduce some $q$-notations and results that will be useful in the next sections. In Sect. 3, we study some spectral properties of the $q$-problem (1.1)-(1.3) by the theory of $q$-(basic) Sturm-Liouville problems [6]. Finally, in Sect. 4, we prove the existence of a spectral function for singular $q$-Dirac system (1.1), and a Parseval's equality is established for vector functions in a Hilbert space.

\section{$2 q$-notations and results}

In this section, we introduce some the required $q$-notations and $q$-results which will be used throughout the paper. Hereafter, $q \in(0,1)$ is fixed (for some details, see [6]). We start with the $q$-shifted factorial for $\alpha \in \mathbb{R}$ and $n=1,2,3, \ldots$ :

$$
(\alpha ; q)_{n}:=\prod_{k=0}^{n-1}\left(1-\alpha q^{k}\right) .
$$

A set $A \subseteq \mathbb{R}$ is called a q-geometric set if $q x \in A$ for all $x \in A$.

The $q$-analogous of sine and cosine functions $[4,6,13]$ are defined on $\mathbb{C}$ by

$$
\begin{aligned}
& \sin (x ; q):=\sum_{m=0}^{\infty} \frac{(-1)^{m} q^{m(m+1)}(x(1-q))^{2 m+1}}{(q ; q)_{2 m+1}} \\
& \cos (x ; q):=\sum_{m=0}^{\infty} \frac{(-1)^{m} q^{m^{2}}(x(1-q))^{2 m}}{(q ; q)_{2 m}} .
\end{aligned}
$$

Let $f$ be a real- or complex-valued function defined on a $q$-geometric set $A$. The $q$ difference operator $D_{q}$, the Jackson q-derivative, is defined by

$$
D_{q} f(x):=\frac{f(x)-f(q x)}{x-q x}, \quad x \in A \backslash\{0\} .
$$

If $0 \in A$, the $q$-derivative at zero is defined by

$$
D_{q} f(0):=\lim _{m \rightarrow \infty} \frac{f\left(x q^{m}\right)-f(0)}{x q^{m}}, \quad x \in A,
$$

if the limit exists and does not depend on $x$. Hence, for $x \in A$,

$$
D_{q^{-1}} f(x)=\left(D_{q} f\right)\left(x q^{-1}\right) .
$$

In the $q$-derivative, as $q \rightarrow 1$, the $q$-derivative is reduced to the classical derivative. 
The right-inverse to $D_{q}$, the Jackson q-integration [20], is given by

$$
\int_{0}^{x} f(t) d_{q} t:=x(1-q) \sum_{m=0}^{\infty} q^{m} f\left(x q^{m}\right), \quad x \in A,
$$

provided that the series converges, and

$$
D_{q} \int_{0}^{x} f(t) d_{q} t=f(x)
$$

moreover, if $\lim _{m \rightarrow \infty} f\left(x q^{m}\right)=f(0)$ for all $x \in A$ (in this case, we say $f$ is $q$-regular at zero), then

$$
\int_{0}^{x} D_{q} f(t) d_{q} t=f(x)-f(0)
$$

Hahn [17] defined the $q$-integration for a function $f$ over $[0, \infty)$ by

$$
\int_{0}^{\infty} f(t) d_{q} t=(1-q) \sum_{m=-\infty}^{\infty} q^{m} f\left(q^{m}\right) .
$$

Furthermore, the following non-symmetric Leibniz formula holds:

$$
D_{q}(f g)(x)=g(x) D_{q} f(x)+f(q x) D_{q} g(x) .
$$

If $f$ and $g$ are $q$-regular at zero, we get

$$
\int_{0}^{a} g(x) D_{q} f(x) d_{q} x=(f g)(a)-(f g)(0)-\int_{0}^{a} f(q x) D_{q} g(x) d_{q} x
$$

The $q$-Wronskian of $f(x)$ and $g(x)$ is defined to be

$$
W_{q}(f, g)(x):=f(x) D_{q} g(x)-g(x) D_{q} f(x), \quad x \in A .
$$

$\left\{Y_{1}, Y_{2}\right\}$ forms a fundamental set of solutions for (1.1) if their $q$-Wronskian does not vanish at any point of $A$.

For more details of $q$-calculus, we also refer the reader to Refs. [5, 7, 13, 24].

\section{Spectral properties of the $q$-Dirac problem}

In this section, we investigate some spectral properties of the $q$-Dirac problem (1.1)-(1.3). Further, the integral equations corresponding to the solution of (1.1) are presented.

Theorem 3.1 The vector eigenfunctions $Y\left(x, \lambda_{1}\right), Y\left(x, \lambda_{2}\right)$ corresponding to the different eigenvalues $\lambda_{1}, \lambda_{2}$ are orthogonal.

Proof Since the vector eigenfunctions

$$
Y\left(x, \lambda_{1}\right)=\left(Y_{1}\left(x, \lambda_{1}\right), Y_{2}\left(x, \lambda_{1}\right)\right), \quad Y\left(x, \lambda_{2}\right)=\left(Y_{1}\left(x, \lambda_{2}\right), Y_{2}\left(x, \lambda_{2}\right)\right)
$$


satisfy the $q$-system (1.1), then

$$
\begin{aligned}
& \frac{1}{q} D_{q} Y_{2}\left(x, \lambda_{1}\right)+p(x) Y_{1}\left(x, \lambda_{1}\right)=\lambda_{1} Y_{1}\left(x, \lambda_{1}\right), \\
& -D_{q^{-1}} Y_{1}\left(x, \lambda_{1}\right)+r(x) Y_{2}\left(x, \lambda_{1}\right)=\lambda_{1} Y_{2}\left(x, \lambda_{1}\right), \\
& \frac{1}{q} D_{q} Y_{2}\left(x, \lambda_{2}\right)+p(x) Y_{1}\left(x, \lambda_{2}\right)=\lambda_{2} Y_{1}\left(x, \lambda_{2}\right), \\
& -D_{q^{-1}} Y_{1}\left(x, \lambda_{2}\right)+r(x) Y_{2}\left(x, \lambda_{2}\right)=\lambda_{2} Y_{2}\left(x, \lambda_{2}\right) .
\end{aligned}
$$

Multiplying (3.1)-(3.4) by $Y_{1}\left(x, \lambda_{2}\right), Y_{2}\left(x, \lambda_{2}\right),-Y_{1}\left(x, \lambda_{1}\right)$ and $-Y_{2}\left(x, \lambda_{1}\right)$, respectively, and applying (2.1) we have

$$
\begin{aligned}
& D_{q}\left(Y_{2}\left(x, \lambda_{1}\right) Y_{1}\left(x q^{-1}, \lambda_{2}\right)-Y_{1}\left(x q^{-1}, \lambda_{1}\right) Y_{2}\left(x, \lambda_{2}\right)\right) \\
& \quad=\left(\lambda_{1}-\lambda_{2}\right)\left(Y_{1}\left(x, \lambda_{1}\right) Y_{1}\left(x, \lambda_{2}\right)+Y_{2}\left(x, \lambda_{1}\right) Y_{2}\left(x, \lambda_{2}\right)\right) .
\end{aligned}
$$

Thus, for each $n \in \mathbb{N}$,

$$
\begin{aligned}
& \int_{0}^{q^{-n}} D_{q}\left(Y_{2}\left(x, \lambda_{1}\right) Y_{1}\left(x q^{-1}, \lambda_{2}\right)-Y_{1}\left(x q^{-1}, \lambda_{1}\right) Y_{2}\left(x, \lambda_{2}\right)\right) d_{q} x \\
& \quad=\left(\lambda_{1}-\lambda_{2}\right) \int_{0}^{q^{-n}} Y\left(x, \lambda_{1}\right) Y^{T}\left(x, \lambda_{2}\right) d_{q} x
\end{aligned}
$$

where $T$ is the transposition sign. This together with (2.2) yields

$$
\begin{aligned}
& \left.\left(Y_{2}\left(x, \lambda_{1}\right) Y_{1}\left(x q^{-1}, \lambda_{2}\right)-Y_{1}\left(x q^{-1}, \lambda_{1}\right) Y_{2}\left(x, \lambda_{2}\right)\right)\right|_{x=0} ^{q^{-n}} \\
& =\left(\lambda_{1}-\lambda_{2}\right) \int_{0}^{q^{-n}} Y\left(x, \lambda_{1}\right) Y^{T}\left(x, \lambda_{2}\right) d_{q} x
\end{aligned}
$$

therefore, from (1.2)-(1.3) we obtain

$$
\left(\lambda_{1}-\lambda_{2}\right) \int_{0}^{q^{-n}} Y\left(x, \lambda_{1}\right) Y^{T}\left(x, \lambda_{2}\right) d_{q} x=0, \quad \forall n \in \mathbb{N},
$$

since $\lambda_{1} \neq \lambda_{2}$, consequently, $Y\left(x, \lambda_{1}\right)$ and $Y\left(x, \lambda_{2}\right)$ are orthogonal.

Theorem 3.2 The eigenvalues of the q-Dirac problem (1.1)-(1.3) are real.

Proof Suppose, on the contrary that $\lambda^{0}$ is a non-real eigenvalue of (1.1)-(1.3), and $Y\left(x, \lambda^{0}\right)$ is the corresponding vector eigenfunction of $\lambda^{0}$. Then $\overline{Y\left(x, \lambda^{0}\right)}$ is the corresponding vector eigenfunction of $\overline{\lambda^{0}}$. Hence, it follows from $\lambda^{0} \neq \overline{\lambda^{0}}$ and (3.5) with $\lambda_{1}=\lambda^{0}, \lambda_{2}=\overline{\lambda^{0}}$ that

$$
\int_{0}^{q^{-n}}\left(\left|Y_{1}\left(x, \lambda^{0}\right)\right|^{2}+\left|Y_{2}\left(x, \lambda^{0}\right)\right|^{2}\right) d_{q} x=0
$$

i.e., $Y\left(x, \lambda^{0}\right) \equiv 0$. This contradiction proves the theorem. 
For each $n \in \mathbb{N}$, the characteristic function for the problem (1.1)-(1.3) is defined by

$$
\Delta_{n}(\lambda):=Y_{1}\left(q^{-n-1}, \lambda\right)-Y_{2}\left(q^{-n}, \lambda\right)
$$

Let $\zeta(x, \lambda)=\left(\zeta_{1}(x, \lambda), \zeta_{2}(x, \lambda)\right)$ be the unique solution [2] of the $q$-Dirac system (1.1) under the initial conditions

$$
\zeta_{1}(0, \lambda)=1=\zeta_{2}(0, \lambda)
$$

Obviously, $\zeta(x, \lambda)$ satisfies (1.2). In the following lemma, we present the integral equations corresponding to the solution $\zeta(x, \lambda)$.

Lemma 3.3 For the solution $\zeta(x, \lambda)$, the following integral equations hold:

$$
\begin{aligned}
\zeta_{1}(x, \lambda) & \\
= & \cos (\lambda \sqrt{q} x ; q)-\frac{1}{\sqrt{q}} \sin (\lambda \sqrt{q} x ; q) \\
& +\sqrt{q} \int_{0}^{x}\{\cos (\lambda \sqrt{q} t ; q) \sin (\lambda \sqrt{q} x ; q)-\sin (\lambda \sqrt{q} t ; q) \cos (\lambda \sqrt{q} x ; q)\} r(t) \zeta_{1}(t, \lambda) d_{q} t \\
& -\int_{0}^{x}\{\cos (\lambda q t ; q) \cos (\lambda \sqrt{q} x ; q)+\sqrt{q} \sin (\lambda q t ; q) \sin (\lambda \sqrt{q} x ; q)\} p(q t) \zeta_{2}(q t, \lambda) d_{q} t, \\
\zeta_{2}(x, & \lambda) \\
= & q \sin (\lambda x ; q)+\cos (\lambda x ; q) \\
& -q \int_{0}^{x}\{\cos (\lambda x ; q) \cos (\lambda \sqrt{q} t ; q)+\sqrt{q} \sin (\lambda x ; q) \sin (\lambda \sqrt{q} t ; q)\} r(t) \zeta_{1}(t, \lambda) d_{q} t \\
& +q \int_{0}^{x}\{\cos (\lambda x ; q) \sin (\lambda q t ; q)-\sin (\lambda x ; q) \cos (\lambda q t ; q)\} p(q t) \zeta_{2}(q t, \lambda) d_{q} t .
\end{aligned}
$$

Proof For $p(x)=r(x)=0$, the $q$-system (1.1) has two solutions

$$
\begin{aligned}
& \psi_{1}(x, \lambda)=(\cos (\lambda \sqrt{q} x ; q), q \sin (\lambda x ; q)), \\
& \psi_{2}(x, \lambda)=(-\sqrt{q} \sin (\lambda \sqrt{q} x ; q), q \cos (\lambda x ; q)),
\end{aligned}
$$

with the $q$-Wronskian

$$
W_{q}\left(\psi_{1}, \psi_{2}\right)(x, \lambda)=q .
$$

Therefore, in the case when $p(x)=r(x)=0$,

$$
\zeta_{g}(x, \lambda)=\left[\begin{array}{c}
c_{1} \cos (\lambda \sqrt{q} x ; q)-c_{2} \sqrt{q} \sin (\lambda \sqrt{q} x ; q) \\
c_{1} q \sin (\lambda x ; q)+c_{2} q \cos (\lambda x ; q)
\end{array}\right]^{T}
$$


is a fundamental set of (1.1). Moreover, a particular solution $\zeta_{p}(x, \lambda)=\left(\zeta_{1}(x, \lambda), \zeta_{2}(x, \lambda)\right)$ of the $q$-system (1.1) may be written as

$$
\left\{\begin{array}{l}
\zeta_{1}(x, \lambda)=v_{1}(x) \cos (\lambda \sqrt{q} x ; q)-v_{2}(x) \sqrt{q} \sin (\lambda \sqrt{q} x ; q) \\
\zeta_{2}(x, \lambda)=v_{1}(x) q \sin (\lambda x ; q)+v_{2}(x) q \cos (\lambda x ; q)
\end{array}\right.
$$

by $q$-analog of the method of variation of parameters, where $v_{1}(x), v_{2}(x)$ are $q$-regular at zero. Substituting (3.10) into (1.1), we obtain the following system:

$$
\left\{\begin{array}{l}
\cos \left(\lambda q^{-\frac{1}{2}} x ; q\right) D_{q^{-1}} v_{1}(x)-\sqrt{q} \sin \left(\lambda q^{-\frac{1}{2}} x ; q\right) D_{q^{-1}} v_{2}(x)=-q p(x) \zeta_{2}(x, \lambda), \\
q \sin (\lambda x ; q) D_{q^{-1}} v_{1}(x)+q \cos (\lambda x ; q) D_{q^{-1}} v_{2}(x)=-q r\left(x q^{-1}\right) \zeta_{1}(x, \lambda),
\end{array}\right.
$$

and hence by Cramer's rule and applying (3.8) we have

$$
\left\{\begin{array}{l}
D_{q^{-1}} v_{1}(x)=-q p(x) \cos (\lambda x ; q) \zeta_{2}(x, \lambda)-r\left(x q^{-1}\right) \sqrt{q} \sin \left(\lambda q^{-\frac{1}{2}} x ; q\right) \zeta_{1}\left(x q^{-1}, \lambda\right), \\
D_{q^{-1}} v_{2}(x)=q p(x) \sin (\lambda x ; q) \zeta_{2}(x, \lambda)-r\left(x q^{-1}\right) \cos \left(\lambda q^{-\frac{1}{2}} x ; q\right) \zeta_{1}\left(x q^{-1}, \lambda\right) .
\end{array}\right.
$$

Since $D_{q^{-1}} v_{i}(x)=\left(D_{q} v_{i}\right)\left(x q^{-1}\right)$, it follows from replacing $x$ by $x q$ in (3.11) and integrating from 0 to $x$ that

$$
\left\{\begin{aligned}
v_{1}(x)= & c_{1}-\sqrt{q} \int_{0}^{x} r(t) \sin (\lambda \sqrt{q} t ; q) \zeta_{1}(t, \lambda) d_{q} t \\
& -q \int_{0}^{x} p(q t) \cos (\lambda q t ; q) \zeta_{2}(q t, \lambda) d_{q} t \\
v_{2}(x)= & c_{2}-\int_{0}^{x} r(t) \cos (\lambda \sqrt{q} t ; q) \zeta_{1}(t, \lambda) d_{q} t \\
& +q \int_{0}^{x} p(q t) \sin (\lambda q t ; q) \zeta_{2}(q t, \lambda) d_{q} t .
\end{aligned}\right.
$$

Now, from (3.9) and (3.12), we can write the general solution of (1.1) as

$$
\begin{aligned}
\zeta_{1}(x, \lambda)= & 2 c_{1} \cos (\lambda \sqrt{q} x ; q)-2 c_{2} \sqrt{q} \sin (\lambda \sqrt{q} x ; q) \\
& +\sqrt{q} \int_{0}^{x}\{\cos (\lambda \sqrt{q} t ; q) \sin (\lambda \sqrt{q} x ; q) \\
& -\sin (\lambda \sqrt{q} t ; q) \cos (\lambda \sqrt{q} x ; q)\} r(t) \zeta_{1}(t, \lambda) d_{q} t \\
& -\int_{0}^{x}\{\cos (\lambda q t ; q) \cos (\lambda \sqrt{q} x ; q) \\
& +\sqrt{q} \sin (\lambda q t ; q) \sin (\lambda \sqrt{q} x ; q)\} p(q t) \zeta_{2}(q t, \lambda) d_{q} t \\
\zeta_{2}(x, \lambda)= & 2 c_{1} q \sin (\lambda x ; q)+2 c_{2} q \cos (\lambda x ; q) \\
& -q \int_{0}^{x}\{\cos (\lambda \sqrt{q} t ; q) \cos (\lambda x ; q)+\sqrt{q} \sin (\lambda \sqrt{q} t ; q) \sin (\lambda x ; q)\} r(t) \zeta_{1}(t, \lambda) d_{q} t \\
& +q \int_{0}^{x}\{\sin (\lambda q t ; q) \cos (\lambda x ; q) \\
& -\cos (\lambda q t ; q) \sin (\lambda x ; q)\} p(q t) \zeta_{2}(q t, \lambda) d_{q} t .
\end{aligned}
$$

Using (3.7), (3.13) and (3.14) we obtain $c_{1}=\frac{1}{2}, c_{2}=\frac{1}{2 q}$, and then the proof is complete. 
In the following theorem, we prove another property of the eigenvalues of (1.1)-(1.3).

Theorem 3.4 The eigenvalues of the problem (1.1)-(1.3) are simple.

Proof The eigenvalues of (1.1)-(1.3) are the zeros of $\Delta_{n}(\lambda)$. From (3.6) we have

$$
\frac{\partial \Delta_{n}(\lambda)}{\partial \lambda}=\frac{\partial Y_{1}\left(q^{-n-1}, \lambda\right)}{\partial \lambda}-\frac{\partial Y_{2}\left(q^{-n}, \lambda\right)}{\partial \lambda}, \quad n \in \mathbb{N}
$$

Now, let $\lambda=\lambda^{0}$ be a double eigenvalue of (1.1)-(1.3) with the corresponding vector eigenfunction $Y\left(x, \lambda^{0}\right)$. Then $\Delta_{n}\left(\lambda^{0}\right)=0$ and $\frac{\partial \Delta_{n}}{\partial \lambda}\left(\lambda^{0}\right)=0$, i.e., for $n \in \mathbb{N}$, the system

$$
\left\{\begin{array}{l}
a Y_{1}\left(q^{-n-1}, \lambda^{0}\right)+b Y_{2}\left(q^{-n}, \lambda^{0}\right)=0, \\
a \frac{\partial Y_{1}}{\partial \lambda}\left(q^{-n-1}, \lambda^{0}\right)+b \frac{\partial Y_{2}}{\partial \lambda}\left(q^{-n}, \lambda^{0}\right)=0
\end{array}\right.
$$

has the nontrivial solution $(a, b)=(1,-1)$. Hence,

$$
Y_{1}\left(q^{-n-1}, \lambda^{0}\right) \frac{\partial Y_{2}}{\partial \lambda}\left(q^{-n}, \lambda^{0}\right)-Y_{2}\left(q^{-n}, \lambda^{0}\right) \frac{\partial Y_{1}}{\partial \lambda}\left(q^{-n-1}, \lambda^{0}\right)=0
$$

On the other hand, differentiating (1.1) with respect to $\lambda$, we get

$$
\left\{\begin{array}{l}
\frac{1}{q} D_{q}\left(\frac{\partial Y_{2}}{\partial \lambda}\right)+(p(x)-\lambda) \frac{\partial Y_{1}}{\partial \lambda}=Y_{1}, \\
-D_{q^{-1}}\left(\frac{\partial Y_{1}}{\partial \lambda}\right)+(r(x)-\lambda) \frac{\partial Y_{2}}{\partial \lambda}=Y_{2} .
\end{array}\right.
$$

Multiplying (1.1) and (3.16) by $\frac{\partial Y_{1}}{\partial \lambda}, \frac{\partial Y_{2}}{\partial \lambda},-Y_{1}$ and $-Y_{2}$, respectively, and applying (2.1), we obtain

$$
\frac{1}{q} D_{q}\left\{Y_{1}\left(x q^{-1}, \lambda\right) \frac{\partial Y_{2}(x, \lambda)}{\partial \lambda}-Y_{2}(x, \lambda) \frac{\partial Y_{1}\left(x q^{-1}, \lambda\right)}{\partial \lambda}\right\}=Y_{1}^{2}(x, \lambda)+\frac{1}{q^{2}} Y_{2}^{2}(x, \lambda) .
$$

Therefore, integrating with respect to $x$ from 0 to $q^{-n}$, with applying (2.2), we have

$$
\begin{gathered}
\left.\frac{1}{q}\left\{Y_{1}\left(x q^{-1}, \lambda\right) \frac{\partial Y_{2}(x, \lambda)}{\partial \lambda}-Y_{2}(x, \lambda) \frac{\partial Y_{1}\left(x q^{-1}, \lambda\right)}{\partial \lambda}\right\}\right|_{x=0} ^{q^{-n}} \\
=\int_{0}^{q^{-n}}\left(Y_{1}^{2}(x, \lambda)+\frac{1}{q^{2}} Y_{2}^{2}(x, \lambda)\right) d_{q} x, \quad n \in \mathbb{N} .
\end{gathered}
$$

According to Lemma 3.3, $\frac{\partial Y_{1}}{\partial \lambda}\left(0, \lambda^{0}\right)=\frac{\partial Y_{2}}{\partial \lambda}\left(0, \lambda^{0}\right)=0$. Taking this and (3.15) into the left-side of (3.17), we obtain

$$
\int_{0}^{q^{-n}}\left(Y_{1}^{2}(x, \lambda)+\frac{1}{q^{2}} Y_{2}^{2}(x, \lambda)\right) d_{q} x=0, \quad n \in \mathbb{N} .
$$

Consequently, $Y_{1}\left(x, \lambda^{0}\right)=Y_{2}\left(x, \lambda^{0}\right) \equiv 0$, i.e. $Y\left(x, \lambda^{0}\right) \equiv 0$. Thus, we arrive at the contradiction. The proof is complete. 


\section{Spectral function and Parseval's equality}

Let $\lambda_{m, n}, m \geq 0, n \in \mathbb{N}$, be the eigenvalues of the $q$-Dirac problem (1.1)-(1.3) (i.e. the roots of $\left.\Delta_{n}(\lambda)\right)$ with the corresponding eigenfunctions

$$
Y_{m, n}(x)=Y\left(x, \lambda_{m, n}\right)=\left(Y_{1}\left(x, \lambda_{m, n}\right), Y_{2}\left(x, \lambda_{m, n}\right)\right) .
$$

If $f(x)=\left(f_{1}(x), f_{2}(x)\right)$ is a vector function, $f_{1}, f_{2} \in L_{q}^{2}\left(0, q^{-n}\right), n \in \mathbb{N}, Y_{m, n, i}(x)=Y_{i}\left(x, \lambda_{m, n}\right)$, $i=1,2$, and

$$
\alpha_{m, n, i}^{2}=\int_{0}^{q^{-n}} Y_{m, n, i}^{2}(x) d_{q} x, \quad i=1,2
$$

then from [7] we have

$$
\int_{0}^{q^{-n}}\left(f_{1}^{2}(x)+f_{2}^{2}(x)\right) d_{q} x=\sum_{m=-\infty}^{\infty} \sum_{i=1}^{2} \frac{1}{\alpha_{m, n, i}^{2}}\left(\int_{0}^{q^{-n}} f_{i}(x) Y_{m, n, i}(x) d_{q} x\right)^{2} .
$$

Denote the non-decreasing step function $\rho_{n}$ by

$$
\rho_{n}(\lambda)= \begin{cases}-\sum_{\lambda<\lambda_{m, n}<0} \sum_{i=1}^{2} \frac{1}{\alpha_{m, n, i}^{2}}, & \lambda<0 \\ \sum_{0 \leq \lambda_{m, n}<\lambda} \sum_{i=1}^{2} \frac{1}{\alpha_{m, n, i}^{2}}, & \lambda \geq 0\end{cases}
$$

Therefore, (4.1) can be written as

$$
\int_{0}^{q^{-n}}\left(f_{1}^{2}(x)+f_{2}^{2}(x)\right) d_{q} x=\int_{-\infty}^{\infty}\left(F_{1, n}^{2}(\lambda)+F_{2, n}^{2}(\lambda)\right) d \rho_{n}(\lambda)
$$

where $F_{i, n}(\lambda)=\int_{0}^{q^{-n}} f_{i}(x) Y_{i}(x, \lambda) d_{q} x, i=1,2$.

Lemma 4.1 For any positive $\tau$, the following inequality holds:

$$
\sum_{-\tau \leq \lambda_{m, n}<\tau} \sum_{i=1}^{2} \frac{1}{\alpha_{m, n, i}^{2}}=\rho_{n}(\tau)-\rho_{n}(-\tau)<\frac{4}{\tau}
$$

Proof Since $\zeta_{1}(x, \lambda)$ and $\zeta_{2}(x, \lambda)$ are continuous at zero, it follows from (3.7) that there is a positive number $\tau$ and nearby zero such that

$$
\frac{1}{\tau} \sum_{i=1}^{2}\left(\int_{0}^{\tau} \zeta_{i}(x, \lambda) d_{q} x\right)^{2}>\frac{1}{2} .
$$

Denote the vector function ${ }_{\tau} f(x)=\left({ }_{\tau} f_{1}(x),{ }_{\tau} f_{2}(x)\right)$ by

$$
{ }_{\tau} f_{i}(x)= \begin{cases}\frac{1}{\tau}, & 0 \leq x \leq \tau \\ 0, & x>\tau\end{cases}
$$


Then, from (4.2) and (4.4), we obtain

$$
\begin{aligned}
\int_{0}^{\tau}\left({ }_{\tau} f_{1}^{2}(x)+{ }_{\tau} f_{2}^{2}(x)\right) d_{q} x & =\frac{2}{\tau}=\int_{-\infty}^{\infty} \sum_{i=1}^{2}\left(\int_{0}^{\tau} \frac{1}{\tau} \zeta_{i}(x, \lambda) d_{q} x\right)^{2} d \rho_{n}(\lambda) \\
& \geq \int_{-\tau}^{\tau} \sum_{i=1}^{2}\left(\frac{1}{\tau} \int_{0}^{\tau} \zeta_{i}(x, \lambda) d_{q} x\right)^{2} d \rho_{n}(\lambda) \\
& >\frac{1}{2}\left(\rho_{n}(\tau)-\rho_{n}(-\tau)\right),
\end{aligned}
$$

and we arrive at (4.3).

The following lemmas were proved in [21].

Lemma 4.2 Let $\left\{v_{n}\right\}_{n=1}^{\infty}$ be a uniformly bounded sequence of real non-decreasing function of $\lambda$ on a finite interval $[a, b]$. Then there exist a subsequence $\left\{v_{n_{k}}\right\}_{k=1}^{\infty}$ and a non-decreasing function $v$ such that, for $\lambda \in[a, b], \lim _{k \rightarrow \infty} v_{n_{k}}(\lambda)=v(\lambda)$.

Lemma 4.3 Assume $\left\{v_{n}\right\}_{n=1}^{\infty}$ is a real uniformly bounded sequence of real non-decreasing function of $\lambda$ on a finite interval $[a, b]$, and suppose for $\lambda \in[a, b], \lim _{n \rightarrow \infty} v_{n}(\lambda)=v(\lambda)$. If $g$ is any continuous function of $\lambda$ on $[a, b]$, then $\lim _{n \rightarrow \infty} \int_{a}^{b} g(\lambda) d v_{n}(\lambda)=\int_{a}^{b} g(\lambda) d v(\lambda)$.

Now, let $\rho$ be any non-decreasing function of $\lambda$ on the interval $(-\infty, \infty)$. We define by $L_{\rho}^{2}(-\infty, \infty) \times L_{\rho}^{2}(-\infty, \infty)$ the Hilbert space of all vector functions $g=\left(g_{1}, g_{2}\right):(-\infty, \infty) \times$ $(-\infty, \infty) \rightarrow \mathbb{R}$ which $g_{1}, g_{2}$ are measurable with respect to the Lebesgue-Stieltjes measure defined by $\rho$, such that $\int_{-\infty}^{\infty} g_{i}(\lambda) d \rho(\lambda)<\infty, i=1,2$, with inner product

$$
\langle g, h\rangle_{\rho}:=\int_{-\infty}^{\infty}\left(g_{1}(\lambda) h_{1}(\lambda)+g_{2}(\lambda) h_{2}(\lambda)\right) d \rho(\lambda) .
$$

In the following theorem, we prove the main result of this section.

Theorem 4.4 For the q-Dirac problem (1.1)-(1.3), there exists a non-decreasing function $\rho(\lambda)$ on the interval $(-\infty, \infty)$ such that satisfies the following property:

If $f=\left(f_{1}, f_{2}\right)$ is a vector function, $f_{i} \in L_{q}^{2}\left(0, q^{-n}\right), i=1,2$, then there exists a function $F=$ $\left(F_{1}, F_{2}\right) \in L_{\rho}^{2}(-\infty, \infty) \times L_{\rho}^{2}(-\infty, \infty)$ such that

$$
\lim _{n \rightarrow \infty} \int_{-\infty}^{\infty}\left\{F_{1}(\lambda)+F_{2}(\lambda)-\int_{0}^{q^{-n}}\left(f_{1}(x) Y_{1}(x, \lambda)+f_{2}(x) Y_{2}(x, \lambda)\right) d_{q} x\right\} d \rho(\lambda)=0
$$

and the Parseval's equality holds:

$$
\int_{0}^{\infty}\left(f_{1}^{2}(x)+f_{2}^{2}(x)\right) d_{q} x=\int_{-\infty}^{\infty}\left(F_{1}^{2}(\lambda)+F_{2}^{2}(\lambda)\right) d \rho(\lambda)
$$

The function $\rho$ is called the spectral function for the q-problem (1.1)-(1.3).

Proof Assume that the vector function $f_{\eta}(x)=\left(f_{\eta, 1}(x), f_{\eta, 2}(x)\right)$ satisfies the following conditions: 
(1) $f_{\eta}(0)=(1,1)$;

(2) $f_{\eta}(x)$ vanishes outside $\left[0, q^{-\eta}\right] \times\left[0, q^{-\eta}\right], q^{-\eta}<q^{-n}$;

(3) $f_{\eta, i}(x)$ and $D_{q} f_{\eta, i}(x), i=1,2$, are $q$-regular at zero.

According to (4.2), we can write

$$
\int_{0}^{q^{-\eta}}\left(f_{\eta, 1}^{2}(x)+f_{\eta, 1}^{2}(x)\right) d_{q} x=\int_{-\infty}^{\infty}\left(F_{1}^{2}(\lambda)+F_{2}^{2}(\lambda)\right) d \rho(\lambda),
$$

where

$$
F_{i}(\lambda)=\int_{0}^{q^{-\eta}} f_{\eta, i}(x) Y_{i}(x, \lambda) d_{q} x, \quad i=1,2 .
$$

Since $Y(x, \lambda)=\left(Y_{1}(x, \lambda), Y_{2}(x, \lambda)\right)$ satisfies the $q$-system (1.1), we have

$$
\left\{\begin{array}{l}
Y_{1}(x, \lambda)=\frac{1}{\lambda}\left(\frac{1}{q} D_{q} Y_{2}(x, \lambda)+p(x) Y_{1}(x, \lambda)\right), \\
Y_{2}(x, \lambda)=\frac{1}{\lambda}\left(-D_{q^{-1}} Y_{1}(x, \lambda)+r(x) Y_{2}(x, \lambda)\right) .
\end{array}\right.
$$

Denote

$$
\begin{aligned}
& F_{n, 1}(\lambda):=\frac{1}{\lambda} \int_{0}^{q^{-n}} f_{\eta, 1}(x)\left(\frac{1}{q} D_{q} Y_{2}(x, \lambda)+p(x) Y_{1}(x, \lambda)\right) d_{q} x, \\
& F_{n, 2}(\lambda):=\frac{1}{\lambda} \int_{0}^{q^{-n}} f_{\eta, 2}(x)\left(-D_{q^{-1}} Y_{1}(x, \lambda)+r(x) Y_{2}(x, \lambda)\right) d_{q} x .
\end{aligned}
$$

Since $f_{\eta}(x)$ vanishes in a neighborhood of $\left(q^{-n}, q^{-n}\right)$, and $f_{\eta}(0)=Y(0, \lambda)=(1,1)$, using $q$ integration by parts we get

$$
\begin{aligned}
& F_{n, 1}(\lambda)=\frac{1}{\lambda} \int_{0}^{q^{-n}}\left\{\frac{1}{q} Y_{2}(x, \lambda) D_{q} f_{\eta, 1}(x)+p(x) f_{\eta, 1}(x) Y_{1}(x, \lambda)\right\} d_{q} x, \\
& F_{n, 2}(\lambda)=\frac{1}{\lambda} \int_{0}^{q^{-n}}\left\{-Y_{1}(x, \lambda) D_{q^{-1}} f_{\eta, 2}(x)+r(x) f_{\eta, 2}(x) Y_{2}(x, \lambda)\right\} d_{q} x .
\end{aligned}
$$

Applying (4.2), we have, for any $\tau>0$,

$$
\begin{aligned}
\int_{|\lambda|>\tau} & F_{n, 1}^{2}(\lambda) d \rho_{n}(\lambda) \\
\leq & \frac{1}{\tau^{2}} \int_{|\lambda|>\tau}\left\{\int _ { 0 } ^ { q ^ { - n } } \left\{\frac{1}{q} Y_{2}(x, \lambda) D_{q} f_{\eta, 1}(x)\right.\right. \\
& \left.\left.+p(x) f_{\eta, 1}(x) Y_{1}(x, \lambda)\right\} d_{q} x\right\}^{2} d \rho_{n}(\lambda) \\
\leq & \frac{1}{\tau^{2}} \int_{-\infty}^{\infty}\left\{\int _ { 0 } ^ { q ^ { - n } } \left\{\frac{1}{q} Y_{2}(x, \lambda) D_{q} f_{\eta, 1}(x)\right.\right. \\
& \left.\left.+p(x) f_{\eta, 1}(x) Y_{1}(x, \lambda)\right\} d_{q} x\right\}^{2} d \rho_{n}(\lambda) \\
= & \frac{1}{\tau^{2}}\left\{\int_{-\infty}^{\infty}\left(\int_{0}^{q^{-n}} \frac{1}{q} Y_{2}(x, \lambda) D_{q} f_{\eta, 1}(x) d_{q} x\right)^{2} d \rho_{n}(\lambda)\right.
\end{aligned}
$$




$$
\left.+\int_{-\infty}^{\infty}\left(\int_{0}^{q^{-n}} Y_{1}(x, \lambda) p(x) f_{\eta, 1}(x) d_{q} x\right)^{2} d \rho_{n}(\lambda)+M_{1}\right\}
$$

where

$$
M_{1}=\frac{2}{q} \int_{-\infty}^{\infty}\left\{\int_{0}^{q^{-n}} Y_{1}(x, \lambda) p(x) f_{\eta, 1}(x) d_{q} x\right\}\left\{\int_{0}^{q^{-n}} Y_{2}(x, \lambda) D_{q} f_{\eta, 1}(x) d_{q} x\right\} d \rho_{n}(\lambda) .
$$

Hence, from (4.2) we obtain

$$
\begin{aligned}
& \int_{|\lambda|>\tau} F_{n, 1}^{2}(\lambda) d \rho_{n}(\lambda) \\
& \quad \leq \frac{1}{\tau^{2}}\left\{\int_{0}^{q^{-n}}\left(p(x) f_{\eta, 1}(x)\right)^{2} d_{q} x+\int_{0}^{q^{-n}}\left(\frac{1}{q} D_{q} f_{\eta, 1}(x)\right)^{2} d_{q} x+M_{1}\right\} .
\end{aligned}
$$

Similarly, we have

$$
\begin{aligned}
& \int_{|\lambda|>\tau} F_{n, 2}^{2}(\lambda) d \rho_{n}(\lambda) \\
& \quad \leq \frac{1}{\tau^{2}}\left\{\int_{0}^{q^{-n}}\left(D_{q^{-1}} f_{\eta, 2}(x)\right)^{2} d_{q} x+\int_{0}^{q^{-n}}\left(r(x) f_{\eta, 2}(x)\right)^{2} d_{q} x+M_{2}\right\},
\end{aligned}
$$

where

$$
M_{2}=-2 \int_{-\infty}^{\infty}\left\{\int_{0}^{q^{-n}} Y_{1}(x, \lambda) D_{q^{-1}} f_{\eta, 2}(x) d_{q} x\right\}\left\{\int_{0}^{q^{-n}} Y_{2}(x, \lambda) r(x) f_{\eta, 2}(x) d_{q} x\right\} d \rho_{n}(\lambda) .
$$

Therefore, it follows from (4.5)-(4.7) that

$$
\begin{aligned}
& \left|\int_{0}^{q^{-\eta}}\left(f_{\eta, 1}^{2}(x)+f_{\eta, 2}^{2}(x)\right) d_{q} x-\int_{-\tau}^{\tau}\left(F_{n, 1}^{2}(\lambda)+F_{n, 2}^{2}(\lambda)\right) d \rho_{n}(\lambda)\right| \\
& \quad=\int_{|\lambda|>\tau}\left(F_{n, 1}^{2}(\lambda)+F_{n, 2}^{2}(\lambda)\right) d \rho_{n}(\lambda) \\
& \quad<\frac{1}{\tau^{2}} \int_{0}^{q^{-\eta}}\left\{\left(p(x) f_{\eta, 1}(x)\right)^{2}+\left(\frac{1}{q} D_{q} f_{\eta, 1}(x)\right)^{2}+\left(D_{q^{-1}} f_{\eta, 2}(x)\right)^{2}\right. \\
& \left.\quad+\left(r(x) f_{\eta, 2}(x)\right)^{2}+M_{1}+M_{2}\right\} d_{q} x .
\end{aligned}
$$

On the other hand, according to Lemma 4.1, the set $\left\{\rho_{n}(\lambda)\right\}$ is bounded. Thus, by Lemmas 4.2 and 4.3 , there is a subsequence $\left\{n_{k}\right\}$ such that $\left\{\rho_{n_{k}}(\lambda)\right\}$ converges to a monotone function $\rho(\lambda)$. Passing to the limit with respect to $\left\{n_{k}\right\}$ in (4.8), we obtain

$$
\begin{aligned}
& \left|\int_{0}^{q^{-\eta}}\left(f_{\eta, 1}^{2}(x)+f_{\eta, 2}^{2}(x)\right) d_{q} x-\int_{-\tau}^{\tau}\left(F_{n, 1}^{2}(\lambda)+F_{n, 2}^{2}(\lambda)\right) d \rho(\lambda)\right| \\
& <\frac{1}{\tau^{2}} \int_{0}^{q^{-\eta}}\left\{\left(p(x) f_{\eta, 1}(x)\right)^{2}+\left(\frac{1}{q} D_{q} f_{\eta, 1}(x)\right)^{2}+\left(D_{q^{-1}} f_{\eta, 2}(x)\right)^{2}\right. \\
& \left.\quad+\left(r(x) f_{\eta, 2}(x)\right)^{2}+M_{1}+M_{2}\right\} d_{q} x .
\end{aligned}
$$


So,

$$
\int_{0}^{q^{-\eta}}\left(f_{\eta, 1}^{2}(x)+f_{\eta, 2}^{2}(x)\right) d_{q} x=\int_{-\infty}^{\infty}\left(F_{n, 1}^{2}(\lambda)+F_{n, 2}^{2}(\lambda)\right) d \rho(\lambda)
$$

as $\tau \rightarrow \infty$. Now, let $f=\left(f_{1}, f_{2}\right)$ be an arbitrary vector function in $L_{q}^{2}(0, \infty) \times L_{q}^{2}(0, \infty)$. We know that there exists a sequence $\left\{f_{\eta}(x)=\left(f_{\eta, 1}(x), f_{\eta, 2}(x)\right)\right\}$ satisfying the conditions (1)-(3) such that

$$
\lim _{\eta \rightarrow \infty} \int_{0}^{\infty}\left(f_{i}(x)-f_{\eta, i}(x)\right)^{2} d_{q} x=0, \quad i=1,2 .
$$

Then

$$
\int_{0}^{\infty}\left(f_{\eta, 1}^{2}(x)+f_{\eta, 2}^{2}(x)\right) d_{q} x=\int_{-\infty}^{\infty}\left(F_{\eta, 1}^{2}(\lambda)+F_{\eta, 2}^{2}(\lambda)\right) d \rho(\lambda)
$$

where $F_{\eta, i}(\lambda)=\int_{0}^{\infty} f_{\eta, i}(x) Y_{i}(x, \lambda) d_{q} x$. Since for $i=1,2$,

$$
\int_{0}^{\infty}\left(f_{\eta_{1}, i}(x)-f_{\eta_{2}, i}(x)\right)^{2} d_{q} x \rightarrow 0
$$

as $\eta_{1}, \eta_{2} \rightarrow \infty$, we get

$$
\int_{-\infty}^{\infty}\left(F_{\eta_{1}, i}(\lambda)-F_{\eta_{2}, i}(\lambda)\right)^{2} d \rho(\lambda)=\int_{0}^{\infty}\left(f_{\eta_{1}, i}(x)-f_{\eta_{2}, i}(x)\right)^{2} d_{q} x \rightarrow 0, \quad i=1,2,
$$

as $\eta_{1}, \eta_{2} \rightarrow \infty$. This is means that there is a limit vector function $F=\left(F_{1}, F_{2}\right)$ such that by the completeness of the space $L_{\rho}^{2}(-\infty, \infty) \times L_{\rho}^{2}(-\infty, \infty)$,

$$
\int_{0}^{\infty}\left(f_{1}^{2}(x)+f_{2}^{2}(x)\right) d_{q} x=\int_{-\infty}^{\infty}\left(F_{1}^{2}(\lambda)+F_{2}^{2}(\lambda)\right) d \rho(\lambda)
$$

Now, it remains to show that the function $\widetilde{F}_{\eta}(\lambda):=\left(\widetilde{F}_{\eta, 1}, \widetilde{F}_{\eta, 2}\right)$ with

$$
\widetilde{F}_{\eta, i}(\lambda):=\int_{0}^{q^{-\eta}} f_{i}(x) Y_{i}(x, \lambda) d_{q} x
$$

as $\eta \rightarrow \infty$, converges to $F=\left(F_{1}, F_{2}\right)$ in $L_{\rho}^{2}(-\infty, \infty) \times L_{\rho}^{2}(-\infty, \infty)$. For this purpose, assume that $s=\left(s_{1}, s_{2}\right)$ is another function in $L_{q}^{2}(0, \infty) \times L_{q}^{2}(0, \infty)$, and by a similar argument, $S(\lambda)$ is defined by $s$. Clearly,

$$
\int_{0}^{\infty}\left(f_{i}(x)-s_{i}(x)\right)^{2} d_{q} x=\int_{-\infty}^{\infty}\left(F_{i}(\lambda)-S_{i}(\lambda)\right)^{2} d \rho(\lambda), \quad i=1,2 .
$$

For $i=1,2$, set

$$
s_{i}(x)= \begin{cases}f_{i}(x), & x \in\left[0, q^{-\eta}\right], \\ 0, & x \in\left(q^{-\eta}, \infty\right) .\end{cases}
$$


Then

$$
\int_{-\infty}^{\infty}\left(F_{i}(\lambda)-\widetilde{F}_{\eta, i}(\lambda)\right)^{2} d \rho(\lambda)=\int_{q^{-\eta}}^{\infty} f_{i}^{2}(x) d_{q} x, \quad i=1,2,
$$

as $\eta \rightarrow \infty$. Consequently, $\widetilde{F}_{\eta}$ converges to $F$ in $L_{\rho}^{2}(-\infty, \infty) \times L_{\rho}^{2}(-\infty, \infty)$ as $\eta \rightarrow \infty$. This completes the proof.

\section{Acknowledgements}

The author gratefully acknowledges that this research is partially supported by the University of Kashan under grant number 682482/10.

\section{Funding}

Not applicable.

\section{Availability of data and materials}

Not applicable.

\section{Competing interests}

The author declares that they have no competing interests.

\section{Author's contributions}

Author read and approved the final manuscript.

\section{Publisher's Note}

Springer Nature remains neutral with regard to jurisdictional claims in published maps and institutional affiliations.

\section{Received: 1 September 2019 Accepted: 10 December 2019 Published online: 16 December 2019}

\section{References}

1. Aldwoah, K.A., Malinowska, A.B., Torres, D.F.M.: The power quantum calculus and variational problems. Dyn. Contin. Discrete Impuls. Syst., Ser. B, Appl. Algorithms 19, 93-116 (2012)

2. Allahverdiev, B.P., Tuna, H.: One dimensional q-Dirac equation. Math. Methods Appl. Sci. 1, 7287-7306 (2017)

3. Allahverdiev, B.P., Tuna, H.: Dissipative q-Dirac operator with general boundary conditions. Quaest. Math. 41, 239-255 (2018)

4. Andrews, G.E., Askey, R., Roy, R.: Special Functions. Cambridge University Press, Cambridge (1999)

5. Annaby, M.H.: q-Type sampling theorems. Results Math. 44, 214-225 (2003)

6. Annaby, M.H., Mansour, Z.S.: Basic Sturm-Liouville problems. J. Phys. A, Math. Gen. 38, 3775-3797 (2005)

7. Annaby, M.H., Mansour, Z.S.: q-Fractional Calculus and Equations. Springer, Berlin (2012)

8. Annaby, M.H., Mansour, Z.S., Soliman, I.A.: q-Titchmarsh-Weyl theory: series expansion. Nagoya Math. J. 205, 67-118 (2012)

9. Chung, K., Chung, W., Nam, S., Kang, H.: New q-derivative and q-logarithm. Int. J. Theor. Phys. 33, $2019-2029$ (1994)

10. Dirac, P.A.M.: The quantum theory of the electron. Proc. R. Soc. A, Math. Phys. Eng. Sci. 117, 610-624 (1928)

11. Floreanini, R., Vinet, L.: A model for the continuous q-ultraspherical polynomials. J. Math. Phys. 36, 3800-3813 (1995)

12. Floreanini, R., Vinet, L.: More on the $q$-oscillator algebra and $q$-orthogonal polynomials. J. Phys. A, Math. Gen. 28, 287-293 (1995)

13. Gasper, G., Rahman, M.: Basic Hypergeometric Series. Cambridge University Press, New York (1990)

14. Gasymov, M.G., Levitan, B.M.: The inverse problem for a Dirac system. Dokl. Akad. Nauk SSSR 167, $967-970$ (1966)

15. Gray, R.W., Nelson, C.A.: A completeness relation for the $q$-analogue coherent states by $q$-integration. J. Phys. A, Math. Gen. 23, 945-950 (1990)

16. Gulsen, T., Yilmaz, E., Koyunbakan, H.: Inverse nodal problem for p-Laplacian Dirac system. Math. Methods Appl. Sci. 40, 2329-2335 (2016)

17. Hahn, W.: Beiträge zur Theorie der Heineschen Reihen. Math. Nachr. 2, 340-379 (1949) (in German)

18. Hira, F.: Eigenvalues and eigenfunctions of $q$-Dirac system. Preprint

19. Jackson, F.H.: q-Difference equations. Am. J. Math. 32, 305-314 (1910)

20. Jackson, F.H.: On q-definite integrals. Quart. J. Pure Appl. Math. 41, 193-203 (1910)

21. Kolmogorov, A.N., Fomin, S.V.: Introductory Real Analysis. Dover, New York (1970). Translated by R.A. Silverman

22. Levitan, B.M., Sargsjan, I.S.: Sturm-Liouville and Dirac Operators. Nauka, Moscow (1988) (in Russian); English transl, Kluwer Academic Publishers, Dordrecht, 1991

23. Malinowska, A.B., Torres, D.F.M.: The Hahn quantum variational calculus. J. Optim. Theory Appl. 147, 419-442 (2010)

24. Mansour, Z.S.: q-difference equations. MSc Thesis, Faculty of Science, Cairo University (2001)

25. Panakhov, E.S.: Inverse problem for Dirac system in two partially settled spectrum. Vinity 3304, 1-29 (1981)

26. Panakhov, E.S., Yilmaz, E., Koyunbakan, H.: Inverse nodal problem for Dirac operator. World Appl. Sci. J. 11, $906-911$ (2010)

27. Prats, F., Toll, J.S.: Construction of the Dirac equation central potential from phase shifts and bound states. Phys. Rev. $113,363-370(1959)$

28. Suslov, S.K.: Another addition theorem for the q-exponential function. J. Phys. A, Math. Gen. 33, 375-380 (2000) 
29. Tariboon, J., Ntouyas, S.K.: Quantum calculus on finite intervals and applications to impulsive difference equations. Adv. Differ. Equ. 2013, 282 (2013)

30. Wei, Z., Guo, Y., Wei, G.: Incomplete inverse spectral and nodal problems for Dirac operator. Adv. Differ. Equ. 2015, 188 (2015)

Submit your manuscript to a SpringerOpen ${ }^{\circ}$ journal and benefit from:

- Convenient online submission

- Rigorous peer review

- Open access: articles freely available online

- High visibility within the field

- Retaining the copyright to your article

Submit your next manuscript at $\boldsymbol{~ s p r i n g e r o p e n . c o m ~}$ 